\title{
Is there a role for vedolizumab in the treatment of ulcerative colitis and Crohn's disease?
}

This article was published in the following Dove Press journal:

Clinical and Experimental Gastroenterology

22 May 2014

Number of times this article has been viewed

\section{Leah Gilroy \\ Patrick B Allen}

Department of Gastroenterology, Ulster Hospital, Dundonald, Belfast, Northern Ireland
Correspondence: Patrick B Allen Department of Gastroenterology, Ulster Hospital, Dundonald, Belfast, Northern Ireland

Email patrick.allen@setrust.hscni.net

\begin{abstract}
Inflammatory bowel disease (IBD) is an important cause of morbidity and mortality for millions of patients worldwide. Current treatment options include corticosteroids, 5 -aminosalicylates, immunosuppressants, and TNF $\alpha$ antagonists. However, these are frequently ineffective in achieving sustained response and remission over time. At present, gastroenterologists lack safe and effective treatments if patients fail anti-TNF therapy. Vedolizumab is a promising new agent for IBD patients refractory to anti-TNF therapy. Vedolizumab is an integrin antagonist which is thought to act by reducing inflammation by selectively inhibiting leukocyte migration in the gut. Emerging evidence from clinical trials suggests a potential role for vedolizumab in both ulcerative colitis (UC) and Crohn's disease (CD), particularly in patients who have previously failed biological therapy. The safety profile of vedolizumab appears reasonable, possibly because it has a "gut-selective" mode of action, with no reported cases of progressive multifocal leukoencephalopathy, a condition which has been linked to another integrin antagonist, natalizumab. This review discusses the available evidence for integrin antagonists and their potential role in the management of IBD.
\end{abstract}

Keywords: vedolizumab, ulcerative colitis, Crohn's disease, inflammatory bowel disease

\section{Introduction}

Inflammatory bowel disease (IBD) affects approximately 3.6 million individuals in the US and in Europe. ${ }^{1}$ Although the incidence and prevalence of IBD varies geographically, the overall incidence has been increasing worldwide. ${ }^{1,2}$ The cost burden can be substantial, as many patients are diagnosed at a relatively young age and management often requires life-long medical and surgical input. ${ }^{3}$ Current treatment modalities for ulcerative colitis (UC) and Crohn's disease (CD) include 5-aminosalicates, corticosteroids, immunosuppressants (including thiopurines, methotrexate, tacrolimus, cyclosporine, and anti-TNF therapies), and surgery.

Over the last decade, the use of anti-TNF- $\alpha$ medications, such as infliximab, adalimumab, certolizumab pegol, and golimumab, have been used in patients with moderate to severe $\mathrm{CD},{ }^{4-7}$ and in those with acute severe colitis ${ }^{8-11}$ who have failed to respond to corticosteroids. Although anti-TNF $\alpha$ treatments are thought to be effective, in real terms, $40 \%$ of UC patients and $20 \%-40 \%$ of CD patients will fail to respond to infliximab therapy. ${ }^{12,13}$

Adalimumab is indicated for the treatment of moderate-to-severe CD. ${ }^{14}$ Adalimumab has a response rate of between $36 \%-41 \%$ in maintaining remission in $\mathrm{CD}$ patients at 56 weeks when given to those patients who have previously failed infliximab. ${ }^{15}$ It also has a reported failure rate of $72 \%$ in maintaining remission in UC patients at 2 years. ${ }^{16}$ 
Certolizumab pegol has been shown to maintain clinical remission at week 26 in $29 \%$ of patients with moderate-tosevere CD versus $18 \%$ of those treated with placebo. ${ }^{7}$

Despite an initial response to anti-TNF therapy, there are a group of patients, ie, "secondary non-responders", who will lose their ability to respond over time. It has been proposed that the development of endogenous antibodies to these drugs, accelerated drug clearance, and ongoing fibrosis or aberrant immune pathways are responsible for this effect. ${ }^{17,18}$

Despite their clear efficacy, it is thought that between $30 \%-40 \%$ of UC patients and $40 \%$ of CD patients who are treated with anti-TNF therapies will lose response with time, at a rate of around $10 \%-13 \%$ per year. ${ }^{17,19,20}$ In addition, the efficacy of a second anti-TNF therapy is lower in those who have previously received an anti-TNF therapy, when compared to those who were previously anti-TNF naïve. ${ }^{21}$ They can also be associated with significant safety issues, including infusion reactions, increased susceptibility to infection, and worsening of congestive cardiac failure. ${ }^{22}$ Consequently, there is an urgent requirement for alternative treatments in patients who fail to respond to anti-TNF therapies.

A new class of therapy, the integrin inhibitors, is being developed and has shown promising results to date. This group of drugs is thought to target and disrupt the leukocyte adhesion and trafficking systems, thereby reducing inflammation. ${ }^{23}$ Vedolizumab is a "gut selective" integrin antagonist and its potential role in IBD is discussed in this review.

\section{Methodology \\ Search strategy}

We conducted a search using PubMed (1947-present) and Medline (1946-present) using the following keywords: vedolizumab, [MLN002, MLN0002, integrin antagonist], AND UC [ulcerative colitis], CD [Crohn's disease], IBD [inflammatory bowel disease]. Keywords were also exploded and selected from MeSH terms for PubMed. References from relevant articles were also searched manually.

\section{Data analysis}

The level and quality of evidence were determined by the study design, sample size, potential bias, statistical analysis, use of controls, and data collection strategy.

\section{Standard protocol approvals, registrations, and patient consent}

Published data were used for this systematic review; therefore, no ethical approval was sought.

\section{Integrin antagonists for treatment in IBD}

Although the exact cause of IBD remains unknown, recent advances in understanding the pathogenesis of UC and CD have led to greater interest in biologic therapies. The most widely used and effective therapies for IBD are monoclonal antibodies targeted against the pro-inflammatory cytokine TNF $\alpha$; however, a significant number of patients fail to respond or lose response over time to these therapies. This has led to research in understanding alternative pathways involved in the inflammation process so as to provide new and alternative targets for therapies. ${ }^{23}$ The migration of leukocytes and other inflammatory cells into intestinal vasculature and disruption of the intestinal barrier function are important in the pathogenesis of IBD. ${ }^{24}$ The high recruitment of T-cells to the intestinal mucosa and subsequent cytokine production has been shown to be key in the pathogenesis of IBD by affecting the endothelial barrier and inducing cell apoptosis in endothelial cells. ${ }^{25}$ Integrin antagonists are antibody-mediated therapies which aim to block the interaction between leukocytes and endothelial cells, and as a result, disrupt trafficking of T-lymphocytes into the inflamed gut (Figure 1). ${ }^{26,27}$

\section{Natalizumab}

The first of the integrin antagonists to emerge was natalizumab, a monoclonal antibody targeted against the adhesion molecule, alpha 4 integrin. Although first used in the treatment of multiple sclerosis, it was subsequently approved for use in CD in $2008 .^{28}$

The efficacy of natalizumab in moderate-to-severe CD was reported in the ENCORE trial where it achieved a clinical response rate of $48 \%$ at weeks 8 to 12 compared to $32 \%$ in the placebo group $(P<0.001) .{ }^{29}$ The ENACT-2 trial reported that patients who responded to initial treatment with natalizumab were more likely to maintain clinical response (61\% versus [vs] 28\%; $P<0.001)$ and remission (44\% vs $26 \% ; P=0.003$ ) with continued natalizumab treatment when compared with patients receiving placebo maintenance at week $36 .^{30}$

Natalizumab remains a second-line treatment of CD and its use is limited by an associated increased incidence of progressive multifocal leukoencephalopathy (PML), a central demyelinating illness caused by the opportunistic human polyoma John Cunningham (JC) virus. ${ }^{31}$ Natalizumab targets the $\alpha_{4}$ monomer, thereby antagonizing both the $\alpha_{4} \beta_{1}$ and $\alpha_{4} \beta_{7}$ integrins. It is hypothesized that through inhibition of the $\alpha_{4} \beta_{1}$ subunit and its interactions, there is reduced immune surveillance in the central nervous system, and consequently, 


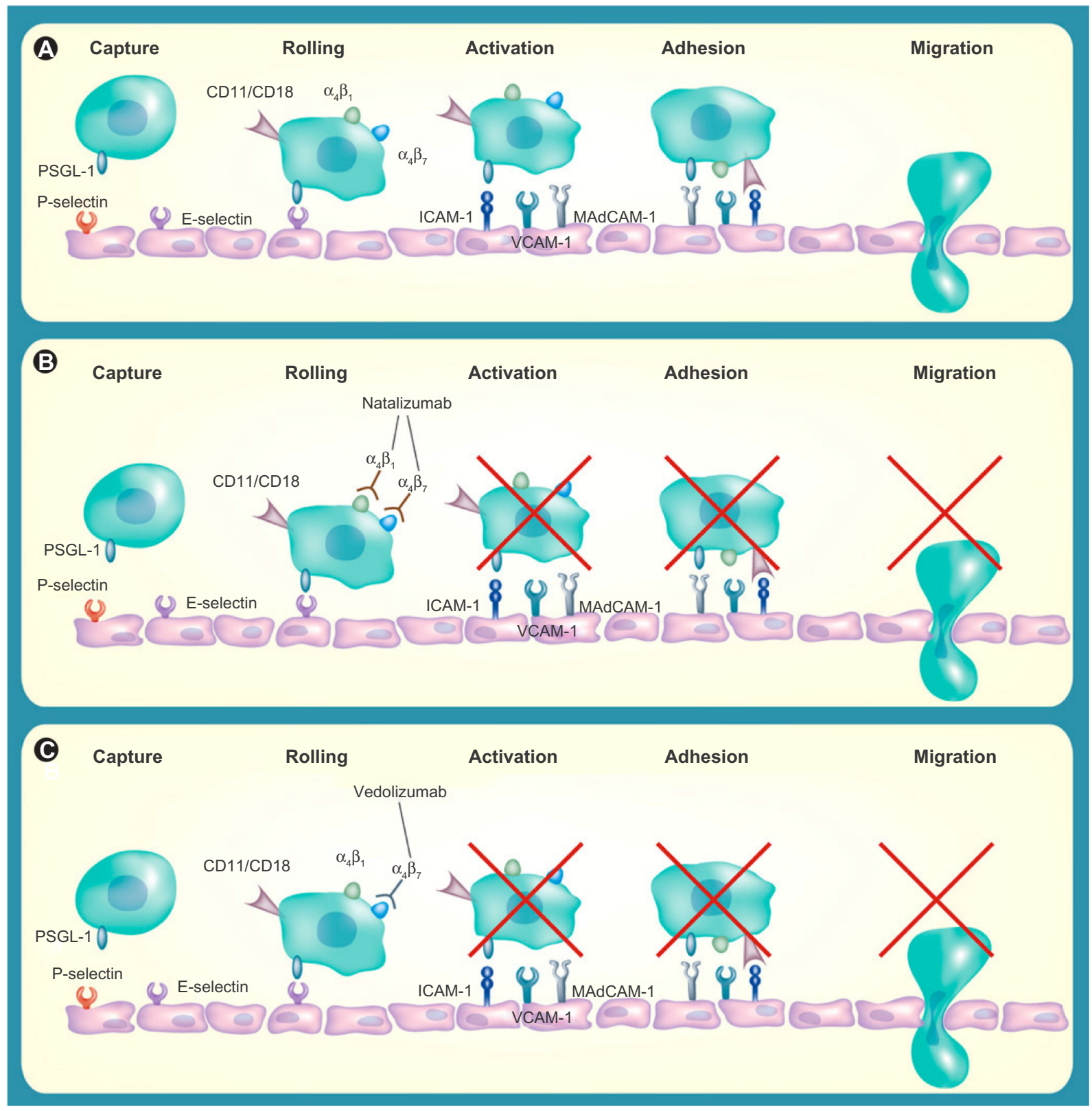

Figure I Blockade of $\alpha$-integrins inhibits leukocyte migration into gut mucosa.

Notes: (A) Tethering/rolling, activation, adhesion, and extravasation/migration of leukocytes into gut mucosa occur through interactions between leukocytes and endothelial cells. (B) Natalizumab prevents leukocyte migration by targeting both the $\alpha_{4} \beta_{1}$ and $\alpha_{4} \beta_{7}$ integrins whereas (C) vedolizumab targets only the $\alpha_{4} \beta_{7}$ integrin, minimizing potential off-target effects such as progressive multifocal leukoencephalopathy, while continuing to inhibit leukocyte migration into gut mucosa. Republished with permission of Future Drugs Ltd, from Fiorino G, Correale C, Fries W, Repici A, Malesci A, Danese S. Leukocyte traffic control: a novel therapeutic strategy for inflammatory bowel disease. Expert Rev Clin Immunol. 20 10;6(4):567-572. ${ }^{67}$ Permission conveyed through Copyright Clearance Center, Inc.

Abbreviation: ICAM-I, Intercellular Adhesion Molecule I; MadCAM-I, mucosal addressin cell adhesion molecule; PSGL, P-selectin glycoprotein ligand; VCAM-I, vascular cell adhesion molecule I.

an increased risk of PML. ${ }^{32}$ The incidence rate has been estimated to be between 0.09 to 11 per 1,000 patients, with higher risk being associated with longer use of natalizumab, prior use of immunosuppressants, and evidence of JC virus infection..$^{33}$ Although natalizumab-associated PML has an improved survival compared with PML in other populations, the mortality rate is up to $29 \%$, with many of the surviving patients suffering from a degree of disability. ${ }^{34}$
Vedolizumab, by selectively targeting the $\alpha_{4} \beta_{7}$ integrin heterodimer, is thought to be "super-selective" and may not cross the blood-brain barrier. ${ }^{35}$ It appears to specifically inhibit lymphocyte migration within the gut. ${ }^{36}$ Studies have reported that it does not affect the levels of T-cells in the cerebrospinal fluid of healthy volunteers after a single dose, and nor does it inhibit immune surveillance of the central nervous system in non-human primates. ${ }^{34,36,37}$ To date, there 
have been no reported cases of PML in patients treated with vedolizumab for UC or CD. ${ }^{38-42}$ The precise targeting of vedolizumab to leukocyte trafficking systems within the gut may provide an improved risk-benefit profile. ${ }^{36,43}$

\section{Vedolizumab}

Vedolizumab (also known as MLN0002, LDP02, and MLN02) is a highly selective monoclonal antibody targeting the $\alpha_{4} \beta_{7}$ integrin molecule. ${ }^{44}$ The $\alpha_{4} \beta_{7}$ integrin is a cell surface glycoprotein variably expressed on lymphocytes and is thought to be partly responsible for T-cell homing into lymphoid tissues in the gastrointestinal tract through its binding to the mucosal addressin cell adhesion molecule (MAdCAM-1). ${ }^{45}$ These bound lymphocytes then migrate from the endothelium of the intestinal vasculature into the lamina propria and tissues, propagating inflammation. ${ }^{46}$ Higher levels of $\alpha_{4} \beta_{7}$ integrin and MAdCAM-1 have been shown to be present in the colons of those with IBD than in patients with irritable bowel syndrome. ${ }^{47}$ It is also thought that there are lower numbers of T-lymphocytes with the $\alpha_{4} \beta_{7}$ integrin circulating in the peripheral blood in patients with colonic inflammation. ${ }^{47}$ As these agents are considered "gut selective", the $\alpha_{4} \beta_{7}$ integrin molecules provide an opportunity to attenuate the pathological gut inflammation seen in patients with IBD. ${ }^{48}$

\section{Vedolizumab: pharmacokinetics and immunogenicity \\ Pharmacokinetics}

During Phase II trials, it was reported that vedolizumab exhibited dose proportional pharmacokinetics, with maximally saturated $\alpha_{4} \beta_{7}$ receptors on peripheral serum lymphocytes over a minimal test dose range of $2 \mathrm{mg} / \mathrm{kg} .{ }^{40}$ As the serum concentration of vedolizumab fell below the level of detection of the assay, $\alpha_{4} \beta_{7}$ integrin-mediated trafficking was restored. The mean elimination half-life of vedolizumab is 15-22 days, with the levels of vedolizumab at one infusion every 8 weeks detectable at a steady level throughout the study, with nearly full inhibition of $\alpha_{4} \beta_{7}$ receptors. ${ }^{40}$

\section{Immunogenicity}

During early Phase II trials of MLN0002, up to 38\% patients developed auto-antibodies. ${ }^{39}$ This unexpectedly high rate of immune sensitization led to an improved formulation of $\alpha_{4} \beta_{7}$ integrin inhibitor to be developed. The new preparation of vedolizumab was developed using the Chinese hamster ovary cell-based system instead of the mouse myeloma cell line that MLN 0002 had been derived from. This new formulation of vedolizumab has been associated with antibody formation in $3.7 \%-4.1 \%$ of patients. ${ }^{49,50}$ The presence of these antibodies is thought to be associated with reduced efficacy of the drug. ${ }^{39,46}$ A dose-related antibody response has also been reported, with a lower proportion of patients who received a higher dose of vedolizumab ( $2 \mathrm{mg} / \mathrm{kg}$ vs $0.5 \mathrm{mg} / \mathrm{kg}$ ) developing antibodies. ${ }^{41}$ The presence of auto-antibodies was also associated with reduced saturation of the $\alpha_{4} \beta_{7}$ binding site. ${ }^{14}$ In a recent Phase III trial, concomitant immunosuppressive therapy was shown to be associated with decreased immunogenicity, which may improve efficacy and increase response rates. ${ }^{49}$

\section{Vedolizumab and UC}

$\mathrm{UC}$ is a chronic condition and can be associated with significant morbidity and disability. ${ }^{51} \mathrm{UC}$ is the most common type of IBD and its frequency in developed countries has been increasing since the mid-20th century. ${ }^{52}$ Approximately $25 \%$ of people with UC will have one or more episodes of acute severe colitis in their lifetime. Although mortality rates have improved steadily over the past 30 years, acute severe colitis still has an average mortality rate of $2 \%$, which can rise to $13 \%$ if an emergency colectomy is required. ${ }^{53,54}$

\section{Vedolizumab in UC: early clinical trials}

The inhibition of $\alpha_{4} \beta_{7}$ integrin was first shown to be effective in inducing remission in colitis in a study on cotton-top tamarin monkeys in $1996 . .^{55}$ The authors reported that there was reduced inflammatory activity and rapidly improved stool consistency when a monoclonal antibody against the $\alpha_{4} \beta_{7}$ integrin was given to eight tamarin monkeys who were chronically affected with colitis. Furthermore, they found that antibody therapy reduced the mucosal density of $\alpha_{4} \beta_{7}$ positive lymphocytes and $\alpha_{4} \beta_{7}$ neutrophils and macrophages. These positive results allowed for vedolizumab to progress to Phase I clinical trials. Table 1 provides a summary of results for vedolizumab.

\section{Vedolizumab in UC: Phase I trial}

In 2000, a double-blind, placebo-controlled Phase I trial using a humanized $\alpha_{4} \beta_{7}$ antibody was carried out in 29 patients with moderate-to-severe UC. ${ }^{38,46}$ The inclusion criteria were that patients had a modified Baron grade ${ }^{56}$ of 2 and a Mayo score ${ }^{57}$ of 5 or more, endoscopic evidence of UC for at least $25 \mathrm{~cm}$ from anal verge and had at least three bowel movements a day. A single dose of the humanized antibody was given to participants in an ascending dose, $0.15 \mathrm{mg} / \mathrm{kg}$ subcutaneous injection (SC), $0.15 \mathrm{mg} / \mathrm{kg}$ intravenous injection (IV), $0.5 \mathrm{mg} / \mathrm{kg} \mathrm{IV}$, 


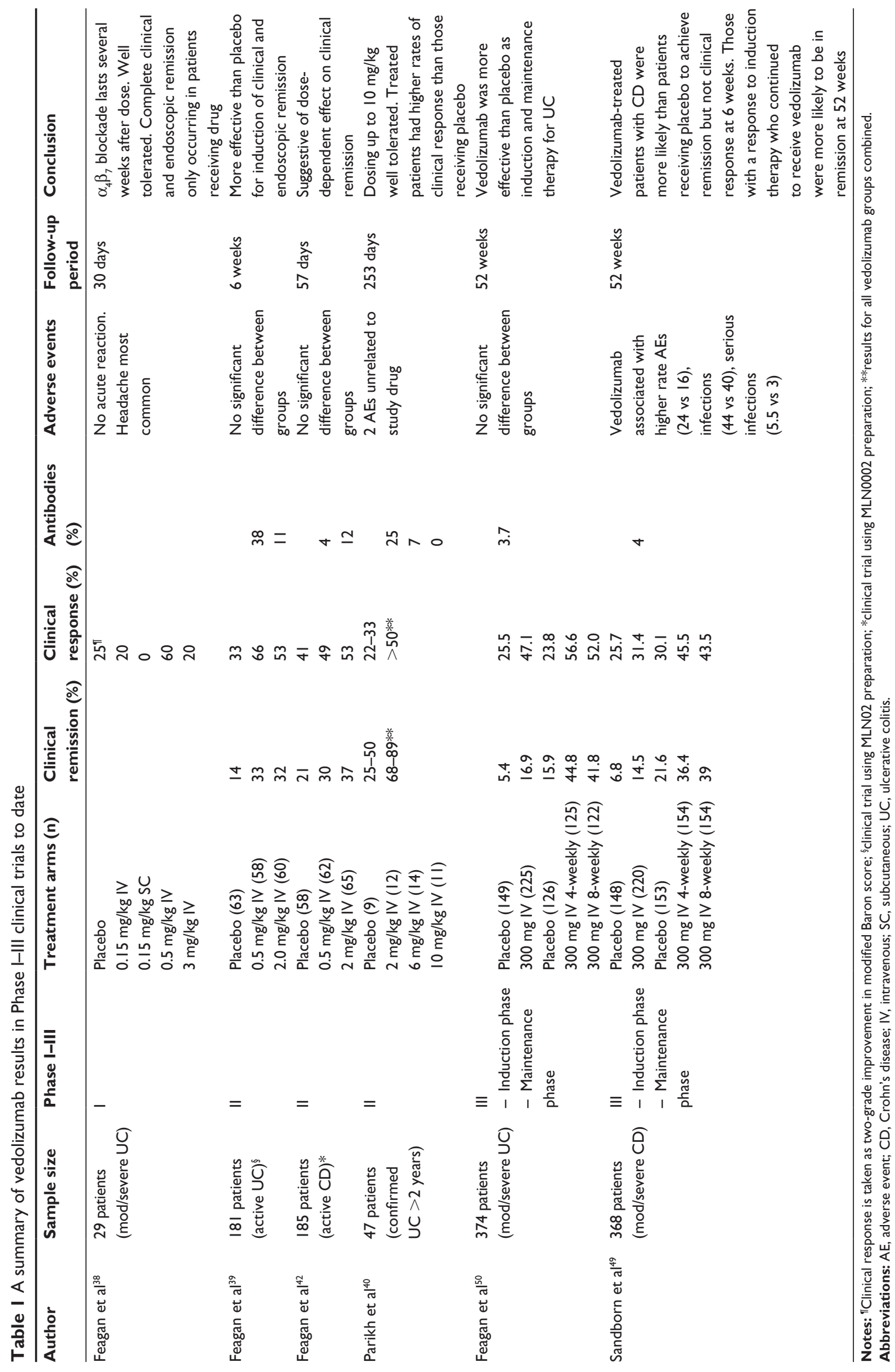


and $3 \mathrm{mg} / \mathrm{kg}$ IV or placebo. A dose of $0.5 \mathrm{mg} / \mathrm{kg}$ IV was found to be enough to give an endoscopic response at day 30 , a two grade improvement in the modified Baron score, and to completely saturate the antibody receptors. Complete endoscopic and clinical remission was seen in $40 \%$ of those patients receiving $0.5 \mathrm{mg} / \mathrm{kg}$ IV. These promising results led to several Phase II trials being commissioned.

\section{Vedolizumab in UC: Phase II trials}

A Phase II trial of $\alpha_{4} \beta_{7}$ antibody (MLN002) was subsequently carried out in 2005 on patients with active UC, a multi-center, double-blind, placebo-controlled trial involving 181 patients. ${ }^{39}$ Patients were randomized to receive either $0.5 \mathrm{mg} / \mathrm{kg}, 2 \mathrm{mg} / \mathrm{kg}$ of MLN002, or placebo intravenously on day 1 and day 29. The authors reported that vedolizumab was significantly more likely to induce clinical remission at 6 weeks compared with placebo. Clinical remission rates (defined as a UC clinical score of $0-1$ and a modified Baron grade of $0-1$ with no evidence of rectal bleeding) at week 6 were $33 \%, 32 \%$, and $14 \%$ for the groups receiving $0.5 \mathrm{mg} / \mathrm{kg}, 2.0 \mathrm{mg} / \mathrm{kg}$, and placebo, respectively ( $P=0.03)$. The corresponding proportion of patients who improved by at least three points on the UC clinical score was also significant between the MLN002 groups ( $66 \%$ in the $0.5 \mathrm{mg} / \mathrm{kg}$, $53 \%$ in the $2 \mathrm{mg} / \mathrm{kg}$ ) and the placebo group (33\%, $P=0.002)$. Furthermore, $28 \%$ of patients receiving $0.5 \mathrm{mg} / \mathrm{kg}$ and $12 \%$ of those receiving $2.0 \mathrm{mg} / \mathrm{kg}$ had endoscopically evident remission, as compared with $8 \%$ of those receiving placebo $(P=0.007)$. Both groups had statistically significant results compared to placebo; however, it is unclear why the clinical and endoscopic remission and response rates were higher in the $0.5 \mathrm{mg} / \mathrm{kg}$ dose group than in the $2 \mathrm{mg} / \mathrm{kg}$ group. Although the authors have not provided a hypothesis for this, it is possible that the higher withdrawal rate in the $2 \mathrm{mg} / \mathrm{kg}$ group ( $8 \%$ vs $2 \%$ ) could have influenced results as those who withdrew early were classified as not achieving remission. No important differences were observed among the three groups in the reasons for withdrawal.

In 2012, the results of a Phase II dose-ranging study were reported by Parikh et al using an improved formulation of vedolizumab. ${ }^{40}$ The study recruited 46 adults with UC (defined as a partial Mayo score $>1$ ) randomized to receive vedolizumab $(2,6$, or $10 \mathrm{mg} / \mathrm{kg})$ or placebo on days $1,15,29$, and 85 , and were followed until day 253. This trial involved higher doses of vedolizumab and shorter frequency between treatment doses than in previous trials. They reported that the clinical response rate of the combined cohort who received vedolizumab was over $50 \%$ compared to between $22 \%-33 \%$ in those treated with placebo. Vedolizumab was also shown to reduce fecal calprotectin levels as compared to placebo.

\section{Vedolizumab in UC: Phase III trials}

In August 2013, the results of the GEMINI I trial were published. This randomized, double-blinded, Phase III trial studied the efficacy and safety of vedolizumab in patients with moderate-to-severe UC. ${ }^{50,58}$ Feagan et al ${ }^{50}$ reported two integrated trials involving 895 patients, to assess the efficacy of vedolizumab for both induction and maintenance therapy in UC.

Patients who had previously failed first-line treatments, including anti-TNF $\alpha$ therapy, as well as those who were anti-TNF $\alpha$ naïve were eligible for this study. Other eligibility criteria included active UC, a Mayo score of $\geq 6$ and an endoscopic subscore of $\geq 2$ despite glucocorticoids, thiopurines, or anti-TNF $\alpha$. The primary outcome for the induction phase of the trial was clinical response to vedolizumab at week 6. Patients were randomized to receive either vedolizumab $300 \mathrm{mg}$ IV or placebo on days 1 and 15. The authors reported that a significantly greater proportion of patients who received vedolizumab achieved clinical response, remission, and mucosal healing at 6 weeks, compared with placebo. Clinical response was achieved in $47 \%$ of the vedolizumab group compared to $26 \%$ in the placebo group $(P<0.0001)$. Clinical remission was seen in $17 \%$ of the vedolizumab group compared with $5 \%$ of those treated with placebo $(P=0.0009)$. Mucosal healing rates were seen in $41 \%$ of those treated with vedolizumab compared with $25 \%$ of the placebo group ( $P=0.0012$ ). Clinical response and remission rates were higher with vedolizumab treatment among both those with prior anti-TNF failure and those with no prior anti-TNF exposure.

GEMINI I also reported the efficacy and safety of maintenance therapy with vedolizumab in moderate-tosevere UC. ${ }^{50,59}$ Those patients achieving clinical response after induction therapy at 0 and 2 weeks, were randomized to receive vedolizumab $300 \mathrm{mg}$ IV at 4-week intervals, vedolizumab $300 \mathrm{mg}$ IV at 8-week intervals, or placebo for 46 weeks.

The results showed that vedolizumab was associated with significantly higher clinical remission rates than placebo at 52 weeks ( $42 \%$ in the vedolizumab 8-weekly group, $45 \%$ in vedolizumab 4-weekly group, and $16 \%$ in the placebo group; $P<0.0001)$. Vedolizumab was also associated with higher mucosal healing rates $(52 \%$ in the vedolizumab 8-weekly group, 56\% in vedolizumab 4-weekly, and $20 \%$ placebo group; $P<0.0001)$. The number of patients who 
were on concomitant glucocorticoids at 52 weeks was significantly lower in those treated with vedolizumab than in those who received placebo (31\% of the vedolizumab 8 -weekly group, $45 \%$ of vedolizumab 4-weekly group, and $14 \%$ placebo group; $P=0.0120 / P<0.0001$, respectively). No clear differences in efficacy were observed between the two vedolizumab regimens. Clinical response and remission rates were greater with vedolizumab in both anti-TNF therapy naïve patients, as well as those with a history of exposure to anti-TNF $\alpha$ agents.

Taken together, these results demonstrate good response and remission rates, mucosal healing, low rate of immunogenicity, and accepted tolerability and safety for vedolizumab in UC in patients who were naïve and in those who had failed anti-TNF therapy.

\section{Vedolizumab and CD}

$\mathrm{CD}$ is a condition that is characterized by trans-mural inflammation, which can affect any area of the gastrointestinal tract, along with many systemic manifestations. It has a relapse rate of $67 \%$ at 5 years, and $10 \%$ of patients will have chronically active disease. ${ }^{60}$ Although the rate of surgical intervention is falling with the increased use of biologics, the lifetime risk of surgery in $\mathrm{CD}$ patients remains high, with up to $60 \%-70 \%$ of patients requiring surgery, dependent on disease severity and location. ${ }^{61}$ Biologics, such as vedolizumab, may assist to improve response, and remission rates may reduce morbidity, mortality, disability, and the cost burden of CD.

\section{Vedolizumab in CD: Phase II trials}

In 2008, Feagan et al reported the results of a randomized, double-blinded, placebo-controlled Phase II trial, which was conducted to assess the efficacy and safety of vedolizumab (then named MLN0002), in patients with active CD. ${ }^{42}$ Adult patients with $\mathrm{CD}$ of the ileum and/or colon, naïve to biologic therapy, and with a CD Activity Index (CDAI) score of 220-400 at screening, were eligible to participate in this study. ${ }^{62}$ One hundred and eighty-three patients were randomized to receive MLN0002 2.0 mg/kg, MLN0002 $0.5 \mathrm{mg} / \mathrm{kg}$, or placebo by IV infusion on days 1 and 29. The randomization was stratified to include concomitant mesalazine use. The primary outcome measure was clinical response at day 57 , defined as an equal or greater than 70-point decrement in the CDAI score. The authors reported that there was no significant difference in clinical response rates between patients who received vedolizumab and those who received placebo. Clinical response rates at day 57 were $53 \%, 49 \%$, and $41 \%$ in the MLN0002 $2.0 \mathrm{mg} / \mathrm{kg}$, MLN0002 $0.5 \mathrm{mg} / \mathrm{kg}$, and placebo groups, respectively. The proportion of patients achieving a more stringently defined enhanced clinical response $(\geq 100$ point decrement in CDAI from baseline) at day 57 were $47 \%$ and $31 \%$, in the MLN0002 $2.0 \mathrm{mg} / \mathrm{kg}$ and placebo groups respectively $(P=0.05)$. Although there was failure to achieve the primary outcome, the results suggested a possible dosedependent effect of vedolizumab in CD.

\section{Vedolizumab in CD: Phase III trials}

More recently, the results of the Gemini II were published. Sandborn et al reported a randomized, double-blind, placebo-controlled Phase III trial, which assessed the efficacy and safety of vedolizumab as induction and maintenance therapy in moderate-to-severe CD. ${ }^{49}$ Patients were randomized to receive either vedolizumab $300 \mathrm{mg}$ IV or placebo on days 1 and 15. As with GEMINI I, the randomization was stratified to take into account past or concomitant use of glucocorticoids, immunosuppressants, and anti-TNF $\alpha$ agents. The primary outcome was considered to be clinical remission $($ CDAI score $=150$ points $)$ and enhanced clinical response (100 point decrease in CDAI from baseline) at 6 weeks. A secondary outcome was mean change in serum $\mathrm{CRP}$ at 6 weeks in patients with elevated $\mathrm{C}$-reactive protein (CRP) at baseline. ${ }^{63}$

The authors reported that a significantly greater proportion of patients receiving vedolizumab achieved clinical remission at week 6 compared to those receiving placebo ( $15 \%$ in the vedolizumab group compared to $7 \%$ of placebo group, $P=0.0206$ ). However, vedolizumab was not associated with a significant difference in clinical response rates compared with placebo at 6 weeks. In those patients who had elevated baseline CRP levels, there was no significant difference in the mean change of CRP level between the groups. Of the intention-to-treat (ITT) population, 48\% had prior anti-TNF $\alpha$ failure; of these, $55 \%$ were primary failures. In addition, $27 \%$ of the ITT population had failed at least two anti-TNF preparations. Positive trends were observed for clinical remission and enhanced clinical response rates in vedolizumab versus placebo patients, irrespective of prior anti-TNF treatment status.

For the maintenance phase of the trial, patients who had a clinical response with vedolizumab at week 6 were randomly assigned to receive 8-weekly vedolizumab, 4-weekly vedolizumab, or placebo regime for up to 52 weeks. The patients that did not have a clinical response to vedolizumab induction therapy received 4-weekly vedolizumab and were followed up to 52 weeks. The primary endpoint was clinical remission at week 52 . At the end of week $52,39 \%$ of 
patients who received 8-weekly vedolizumab and $36 \%$ of patients receiving 4-weekly vedolizumab were in clinical remission, as compares to $22 \%$ of those who received placebo $(P<0.001$ and $P=0.004$ when comparing each group with placebo, respectively). The proportion of patients who had a 100-point reduction in CDAI score and glucocorticoid-free remission were significantly greater in both vedolizumab groups; however, rates of durable clinical remission showed no significant differences.

\section{Why are there differences in the efficacy of vedolizumab between $U C$ and $C D$ ?}

Response and remission rates for vedolizumab vary between $\mathrm{UC}$ and $\mathrm{CD}$. Clinical response rates in UC were reported between $47 \%-50 \%$, with durable clinical remission rates between $41 \%-45 \%$ and mucosal healing rates of between $28 \%-56 \%{ }^{50,39}$ In CD, the results were less encouraging, with a response rate of $31 \%-53 \%$ and durable clinical remission rates of between $36 \%-39 \% .^{49,42}$ It has been suggested that the difference arises due to the "super-selective" nature of vedolizumab. CD is a systemic disorder, characterized by transmural inflammation which can affect any part of the gastrointestinal tract from mouth to anus, whereas UC is considered a more localized disease associated with sub-mucosal inflammation, more limited to the large bowel. Vedolizumab, due to its specificity for the $\alpha_{4} \beta_{7}$ integrin heterodimer, is thought to exert a localized mode of action. This is in contrast to natalizumab, which has been shown to have good efficacy in CD and inhibits both the $\alpha_{4} \beta_{7}$ and $\alpha_{4} \beta_{1}$, integrins, possibly resulting in a greater systemic blockade on leukocyte trafficking. ${ }^{24}$ Another hypothesis is that vedolizumab may require a longer time to produce a clinical response by inhibiting leukocyte migration in CD than in UC. This was reported in the GEMINI II trial results when vedolizumab failed to achieve its primary endpoint of clinical response at week 6 but showed efficacy at maintaining remission at week $52 .{ }^{49}$ Overall, the evidence of vedolizumab in $\mathrm{CD}$ is promising, yet more data is required to fully characterize which subset of CD patients would derive most benefit.

\section{Safety and tolerability of vedolizumab}

The overall safety profile of vedolizumab throughout the clinical trials conducted has been positive and consistent with the concept of selective immunosuppression. ${ }^{49,64}$ Clinically important infusion reactions were rare. ${ }^{40,49,50}$ Doses of $10 \mathrm{mg} / \mathrm{kg}$ of vedolizumab were well tolerated. ${ }^{40}$ As stated before, no cases of PML have yet been reported despite more than 3,000 patients having been exposed to vedolizumab for periods of up to 6 years. ${ }^{37,39,40,64}$ In the large GEMINI I study, no significant difference was observed among the study groups in the most commonly reported adverse incidents. ${ }^{50}$ The most common adverse advents reported were exacerbation of IBD, headache, and nasopharyngitis. ${ }^{49,50}$ The GEMINI II study, however, found that the incidence of infections and serious infections was higher in those who received vedolizumab than with placebo. They also found that the incidence of any serious event was higher among those who received vedolizumab than among those who received placebo (25\% vs $15 \%$ ). Long term observational data combined with data from Phase IV trials where large numbers of patients are exposed are needed to fully characterize the safety profile of vedolizumab, including the risk, if any, of developing PML. Vedolizumab is currently being assessed in a Phase III trial to determine its long-term safety (GEMINI LTS) and is due to be completed in March 2016. ${ }^{65}$

\section{The future role of vedolizumab in IBD}

Vedolizumab appears to be an important, much needed new therapy for patients with IBD. Standard therapies are suboptimal, with many patients not responding, relapsing, or experiencing common side effects. In UC, vedolizumab has shown efficacy in inducing and maintaining remission in patients who have failed first-line treatment, as well as those who have failed anti-TNF $\alpha$ therapy. Therefore, one important role of vedolizumab may be in refractory UC prior to surgical intervention. Vedolizumab has also been shown to be effective in $\mathrm{CD}$, although more research is required to identify a subset of patients who will achieve the most benefit from its initiation.

In the future, vedolizumab and other disease-modifying drugs may have a place in first-line therapy in those patients with IBD. Current medical management of IBD is based on a step-wise system with treatments being used sequentially and treatment escalated as patients fail to respond to each step of treatment. However, there is mounting evidence that focusing treatment on mucosal healing, rather than clinical symptoms, may result in a reduction in the need for surgery and reduced hospitalization rates for both UC and CD. ${ }^{66}$ Similar to the treatment strategies currently used in rheumatoid arthritis, the elimination of disability and reduction of structural bowel damage has emerged as a new therapeutic goal. ${ }^{13}$ The concept of identifying patients who have a high risk of morbidity early in their disease and introducing disease modifying anti-IBD drugs, including vedolizumab 
and other small molecule inhibitors is growing in favor with gastroenterologists. ${ }^{13}$

To date, vedolizumab has shown a good level of patient acceptability and a reasonable safety profile; however, prospective studies are required to ascertain its long-term benefits and side-effect profile in the treatment of IBD. It seems probable that vedolizumab will become available as a secondline treatment for those patients who fail anti-TNF therapy, especially in UC. However, as more data becomes available, vedolizumab may become the first-line or adjunctive therapy in certain patients with moderate-to-severe IBD.

\section{Disclosure}

The authors report no conflicts of interest in this work.

\section{References}

1. Loftus EV Jr. Clinical epidemiology of inflammatory bowel disease: Incidence, prevalence, and environmental influences. Gastroenterology. 2004;126:1504-1517.

2. Molodecky NA, Soon IS, Rabi DM, et al. Increasing incidence and prevalence of the inflammatory bowel diseases with time, based on systematic review. Gastroenterology. 2011;142:46-54. e42.

3. Park KT, Bass D. Inflammatory bowel disease-attributable costs and cost-effective strategies in the United States: a review. Inflamm Bowel Dis. 2011;17:1603-1609.

4. Sands BE, Anderson FH, Bernstein CN, et al. Infliximab maintenance therapy for fistulizing Crohn's disease. New Eng J Med. 2004;350(9): 876-885.

5. Hanauer SB, Feagan BG, Lichtenstein GR, et al; ACCENT I Study Group. Maintenance infliximab for Crohn's disease: the ACCENT I randomised trial. Lancet. 2002;359(9317):1541-1549.

6. Sandborn WJ, Hanauer SB, Rutgeerts P, et al. Adalimumab for maintenance treatment of Crohn's disease: results of the CLASSIC II trial. Gut. 2007;56(9):1232-1239.

7. Sandborn WJ, Feagan BG, Stoinov S, et al; PRECISE 1 Study Investigators. Certolizumab pegol for the treatment of Crohn's disease. New Eng J Med. 2007;357(3):228-238.

8. Dignass A, Lindsay JO, Sturm A, et al. Second European evidence-based consensus on the diagnosis and management of ulcerative colitis part 2: current management. J Crohns Colitis. 2012;6(10):991-1030.

9. Rutgeerts P, Sandborn WJ, Feagan BG, et al. Infliximab for induction and maintenance therapy for ulcerative colitis. New Eng J Med. 2005;353(23):2462-2476.

10. Reinisch W, Sandborn WJ, Hommes DW, et al. Adalimumab for induction of clinical remission in moderately to severely active ulcerative colitis: results of a randomised controlled trial. Gut. 2011;60(6): 780-787.

11. Sandborn WJ, Feagan BG, Marano C, et al; PURSUIT-Maintenance Study Group. Subcutaneous golimumab maintains clinical response in patients with moderate-to-severe ulcerative colitis. Gastroenterology. 2014;146(1):96-109. e1.

12. Ford AC, Sandborn WJ, Khan KJ, Hanauer SB, Talley NJ, Moayyedi P. Efficacy of biological therapies in inflammatory bowel disease: systematic review and meta-analysis. Am J Gastroenterol. 2011;106(4):644-659, quiz 660.

13. Allen PB, Peyrin-Biroulet L. Moving towards disease modification in inflammatory bowel disease therapy. Curr Opin Gastroenterol. 2013;29(4):397-404

14. McLean LP, Shea-Donohue T, Cross RK. Vedolizumab for the treatment of ulcerative colitis and Crohn's disease. Immunotherapy. 2012;4(9): 883-898.
15. Lichtenstein GR, Panaccione R, Mallarkey G. Efficacy and safety of adalimumab in Crohn's disease. Therap Adv Gastroenterol. 2008;1(1): $43-50$.

16. McDermott E, Murphy S, Keegan D, O’Donoghue D, Mulcahy H, Doherty G. Efficacy of Adalimumab as a long term maintenance therapy in ulcerative colitis. J Crohns Colitis. 2013;7(2):150-153.

17. Ben-Horin S, Chowers Y. Review article: loss of response to anti-TNF treatments in Crohn's disease. Aliment Pharmacol Ther. 2011;33(9): 987-995.

18. Afif W, Loftus EV, Faubion WA, et al. Clinical utility of measuring infliximab and human anti-chimeric antibody concentrations in patients with inflammatory bowel disease. Am J Gastroenterol. 2010;105(5): $1133-1139$.

19. Leung Y, Panaccione R. Anti-adhesion molecule strategies for Crohn disease. Bio Drugs. 2008;22(4):259-264.

20. Gisbert JP, Panés J. Loss of response and requirement of infliximab dose intensification in Crohn's disease: a review. Am J Gastroenterol. 2009;104(3):760-767.

21. Sandborn WJ, Rutgeerts P, Enns R, et al. Adalimumab induction therapy for Crohn disease previously treated with infliximab: a randomized trial. Ann Intern Med. 2007;146(12):829-838.

22. Rutgeerts $P$, van Assche G, Vermeire S. Optimizing anti-TNF treatment in inflammatory bowel disease. Gastroenterology. 2004;126(6): 1593-1610.

23. Ghosh S, Panaccione R. Anti-adhesion molecule therapy for inflammatory bowel disease. Therap Adv Gastroenterol. 2010;3(4):239-258.

24. Cominelli F. Inhibition of leukocyte trafficking in inflammatory bowel disease. New Eng J Med. 2013;369(8):775-776.

25. Fuss IJ, Heller F, Boirivant M, et al. Nonclassical CD1d-restricted NK $\mathrm{T}$ cells that produce IL-13 characterize an atypical Th2 response in ulcerative colitis. J Clin Invest. 2004;113:1490-1497.

26. Bevilacqua MP. Endothelial-leukocyte adhesion molecules. Annu Rev Immunol. 1993;11:767-804.

27. Van Assche G, Rutgeerts P. Physiological basis for novel drug therapies used to treat the inflammatory bowel diseases. I. Immunology and therapeutic potential of antiadhesion molecule therapy in inflammatory bowel disease. Am J Physiol Gastrointest Liver Physiol. 2005;288(2): G169-G174.

28. Holmes D. Integrin inhibitors go with the gut. Nat Rev Drug Discov. 2013;12(6):411-412.

29. Targan SR, Feagan BG, Fedorak RN, et al; International Efficacy of Natalizumab in Crohn's Disease Response and Remission (ENCORE) Trial Group. Natalizumab for the treatment of active Crohn's disease: results of the ENCORE Trial. Gastroenterology. 2007;132(5): $1672-1683$.

30. Sandborn WJ. The future of inflammatory bowel disease therapy: where do we go from here? Dig Dis. 2012;30 Suppl 3:140-144.

31. Danese S. New therapies for inflammatory bowel disease: from the bench to the bedside. Gut. 2012;61(6):918-932.

32. FDA Drug Safety Communication: New risk factor for Progressive Multifocal Leukoencephalopathy (PML) associated with Tysabri (natalizumab) [webpage on the Internet]. Center for Drug Evaluation and Research; 2012 [cited January 20, 2012]. Available from http://www. fda.gov/Drugs/DrugSafety/ucm288186.htm. Accessed October 30, 2013.

33. Bloomgren G, Richman S, Hotermans C, et al. Risk of natalizumabassociated progressive multifocal leukoencephalopathy. New Eng J Med. 2012;366(20):1870-1880.

34. Vermersch P, Kappos L, Gold R, et al. Clinical outcomes of natalizumab-associated progressive multifocal leukoencephalopathy. Neurology. 2011;76(20):1697-1704.

35. Milch C, Wyant T, Xu J, et al. Vedolizumab, a monoclonal antibody to the gut homing $\alpha 4 \beta 7$ integrin, does not affect cerebrospinal fluid T-lymphocyte immunophenotype. J Neuroimmunol. 2013;264(1-2): 123-126.

36. Allen PB. Anti-adhesion molecules: is gut specificity the key for a good safety profile? Curr Drug Deliv. 2012;9:333-337. 
37. Fedyk ER, Wyant T, Yang L-L, et al. Exclusive antagonism of the $\alpha(4) \beta(7)$ integrin by vedolizumab confirms the gut-selectivity of this pathway in primates. Inflamm Bowel Dis. 2012;18:2107-2119.

38. Feagan B, Macdonald J, Greenberg G, et al. An ascending dose of a humanized alpha 4 beta 7 antibody in ulcerative colitis (UC). Gastroenterology. 2000;118(4):A874.21.

39. Feagan BG, Greenberg GR, Wild G, et al. Treatment of ulcerative colitis with a humanized antibody to the alpha4beta7 integrin. New Eng JMed. 2005;352(24):2499-2507.

40. Parikh A, Leach T, Wyant T, et al. Vedolizumab for the treatment of active ulcerative colitis: a randomized controlled phase 2 dose-ranging study. Inflammat Bowel Dis. 2012;18:1470-1479.

41. Parikh A, Leach T, Xu J, Feagan B. Long-term clinical experience with vedolizumab (VDZ) in patients with mild to moderate ulcerative colitis (UC). J Crohns Colitis. 2012;6:S103.

42. Feagan BG, Greenberg GR, Wild G, et al. Treatment of active Crohn's disease with MLN0002, a humanized antibody to the alpha4beta7 integrin. Clin Gastroenterol Hepatol. 2008;6(12):1370-1377.

43. Soler D, Chapman T, Yang L-L, Wyant T, Egan R, Fedyk ER. The binding specificity and selective antagonism of vedolizumab, an anti-alpha4beta7 integrin therapeutic antibody in development for inflammatory bowel diseases. J Pharmacol Exp Ther. 2009;330: 864-875.

44. Marshall JK. LDP-02 (Millenium). Curr Opin Investig Drugs. 2001;2: 502-504.

45. Petrovic A, Alpdogan O, Willis LM, et al. LPAM (alpha 4 beta 7 integrin) is an important homing integrin on alloreactive T cells in the development of intestinal graft-versus-host disease. Blood. 2004;103: 1542-1547

46. Gledhill T, Bodger K. New and emerging treatments for ulcerative colitis: a focus on vedolizumab. Biologics. 2013;7:123-130.

47. Souza HS, Elia CC, Spencer J, MacDonald TT. Expression of lymphocyte-endothelial receptor-ligand pairs, alpha4beta7/MAdCAM-1 and OX40/OX40 ligand in the colon and jejunum of patients with inflammatory bowel disease. Gut. 1999;45:856-863.

48. Meenan J, Spaans J, Grool TA, et al. Altered expression of alpha 4 beta 7 , a gut homing integrin, by circulating and mucosal $\mathrm{T}$ cells in colonic mucosal inflammation. Gut. 1997;40:241-246.

49. Sandborn WJ, Feagan BG, Rutgeerts P, et al; GEMINI 2 Study Group. Vedolizumab as induction and maintenance therapy for Crohn's disease. New Eng J Med. 2013;369(8):711-721.

50. Feagan BG, Rutgeerts P, Sands BE, et al; GEMINI 1 Study Group. Vedolizumab as induction and maintenance therapy for ulcerative colitis. New Eng J Med. 2013;369(8):699-710.

51. Allen PB, Kamm MA, Peyrin-Biroulet L, et al. Development and validation of a patient-reported disability measurement tool for patients with inflammatory bowel disease. Aliment Pharmacol Therap. 2013;37(4): $438-444$.

52. Danese S, Fiocchi C. Ulcerative colitis. New Eng J Med. 2011;365(18): 1713-1725.

53. NICE. Management of ulcerative colitis. NICE Guidelines. Jun 2013. Available from http://publications.nice.org.uk/ulcerative-colitis-cg166. Accessed September 14, 2013.
54. Bitton A, Buie D, Enns R, et al; Canadian Association of Gastroenterology Severe Ulcerative Colitis Consensus Group. Treatment of hospitalized adult patients with severe ulcerative colitis: Toronto consensus statements. Am J Gastroenterol. 2012;107(2):179-194; author reply 195 .

55. Hesterberg PE, Winsor-Hines D, Briskin MJ, et al. Rapid resolution of chronic colitis in the cotton-top tamarin with an antibody to a gut-homing integrin alpha 4 beta 7. Gastroenterology. 1996;111(5):1373-1380.

56. Baron JH, Connell AM, Lennard-Jones JE. Variation between observers in describing mucosal appearances in proctocolitis. $B M J$. 1964;1(5375):89-92.

57. Schroeder KW, Tremaine WJ, Ilstrup DM. Coated oral 5-aminosalicylic acid therapy for mildly to moderately active ulcerative colitis. A randomized study. New Eng J Med. 1987;317(26):1625-1629.

58. Feagan BG, Rutgeerts PJ, Sands BE, et al. 943b Induction therapy for ulcerative colitis: results of GEMINI I, a randomized, placebocontrolled, double-blind, multicenter phase 3 trial. Gastroenterology. 2012;142(5):S160-S161.

59. Rutgeerts PJ. Vedolizumab maintenance therapy for ulcerative colitis (uc): results of GEMINI I, a randomized, placebo-controlled, doubleblind, multicenter phase 3 trial. Gut. 2012;61(supp 3):A65.

60. Lapidus A, Bernell O, Hellers G, Löfberg R. Clinical course of colorectal Crohn's disease: a 35-year follow-up study of 507 patients. Gastroenterology. 1998;114(6):1151-1160.

61. Mowat C, Cole A, Windsor A, et al; IBD Section of the British Society of Gastroenterology. Guidelines for the management of inflammatory bowel disease in adults. Gut. 2011;60(5):571-607.

62. Baumgart DC. Veto on vedolizumab (MLN0002) for Crohn's disease. Inflammat Bowel Dis. 2010;16(3):537-538.

63. Sandborn W, Sands BE, Colombel JF, et al. Vedolizumab induction therapy for Crohns Disease: results of GEMINI II, a randomized, placebo controlled, double-blind, multi-center phase 3 trial. P395. Annual Scientific Meeting Abstracts. 2012. Las Vegas, NV: American College of Gastroenterology.

64. Mosli MH, Feagan BG. Vedolizumab for Crohn's disease. Expert Opin Biol Ther. 2013;13:455-463.

65. Millennium Pharmaceuticals, Inc. An open-label study of vedolizumab (MLN0002) in patients with ulcerative colitis and Crohn's disease. Available from: http://clinicaltrials.gov/show/NCT00790933. NLM identifier: NCT00790933. Accessed October 15, 2013.

66. Peyrin-Biroulet L, Ferrante M, Magro F, et al; Scientific Committee of the European Crohn's and Colitis Organization. Results from the 2nd Scientific Workshop of the ECCO. I: Impact of mucosal healing on the course of inflammatory bowel disease. J Crohns Colitis. 2011;5(5): 477-483.

67. Fiorino G, Correale C, Fries W, Repici A, Malesci A, Danese S. Leukocyte traffic control: a novel therapeutic strategy for inflammatory bowel disease. Expert Rev Clin Immunol. 2010;6(4):567-572.
Clinical and Experimental Gastroenterology

\section{Publish your work in this journal}

Clinical and Experimental Gastroenterology is an international, peerreviewed, open access journal, publishing all aspects of gastroenterology in the clinic and laboratory, including: Pathology, pathophysiology of gastrointestinal disease; Investigation and treatment of gastointestinal disease; Pharmacology of drugs used in the alimentary tract;
Dovepress

Immunology/genetics/genomics related to gastrointestinal disease. This journal is indexed on CAS. The manuscript management system is completely online and includes a very quick and fair peer-review system. Visit http://www.dovepress.com/testimonials.php to read real quotes from published authors. 